\title{
Pulse versus daily oral Alfacalcidol treatment of secondary hyperparathyroidism in hemodialysis patients: a randomized controlled trial
}

This article was published in the following Dove Press journal: International Journal of Nephrology and Renovascular Disease

\author{
Osama Sawalmeh' \\ Shaheed Moala' \\ Zakaria Hamdan² \\ Huda Masri ${ }^{3}$ \\ Khubaib Ayoub ${ }^{4}$ \\ Emad Khazneh ${ }^{2}$ \\ Mujahed Shraim ${ }^{5}$ \\ 'Medicine Department, Faculty \\ of Medicine and Health Sciences, \\ An-Najah National University, Nablus, \\ Palestine; ${ }^{2}$ Nephrology Department, \\ ${ }^{3}$ Pharmacy Department, ${ }^{4}$ Internal \\ Medicine Department, An-Najah \\ National University Hospital, Nablus, \\ Palestine; ${ }^{5}$ Public Health Department, \\ College of Health Sciences, Qatar \\ University, Doha, Qatar
}

Background: Secondary hyperparathyroidism is a common complication of chronic kidney disease and is managed using vitamin D replacement therapy. Very few studies have examined the effectiveness of pulse alfacalcidol therapy in comparison to daily oral alfacalcidol therapy in suppressing serum parathyroid hormone (PTH) levels in hemodialysis patients. The aim of this randomized controlled trial was to replicate the findings of prior studies comparing effectiveness of pulse oral alfacalcidol therapy versus daily oral alfacalcidol therapy in suppressing PTH after 13 weeks of therapy using a Palestinian sample of hemodialysis patients, and to identify demographic and biomedical characteristics of patients that are independently associated with PTH levels

Methods: One hundred and sixty-seven patients completed the study, 88 in the daily group and 79 in the pulse group. The pulse group had more clinically significant reduction in mean PTH level by $75 \mathrm{pg} / \mathrm{dL}$ at 13 weeks than the daily group, but this was not statistically significant.

Results: The effect of alfacalcidol therapy on metabolism of phosphate and corrected calcium levels was comparable in both groups, and pulse therapy was not associated with increased risk of hypercalcemia and hyperphosphatemia. Serum PTH levels were independently and inversely associated with older age and diabetes.

Conclusion: Switching daily alfacalcidol therapy to thrice-weekly alfacalcidol pulse therapy seems safe and convenient, especially for hemodialysis patients with poor compliance with treatment. This study also highlights the importance of monitoring and preventing malnutrition in hemodialysis patients and maintaining optimal glycemic control in diabetic hemodialysis patients. Keywords: end-stage kidney disease, hemodialysis, alfacalcidol, parathyroid hormone, hyperparathyroidism, randomized controlled trials, pulse therapy

\section{Background}

Secondary hyperparathyroidism, characterized by elevated serum parathyroid hormone (PTH), autonomous secretion parathyroid hyperplasia, is a common complication of chronic kidney disease. ${ }^{1,2}$ Progression of secondary hyperparathyroidism in chronic kidney disease is attributed to a combination of factors, including reduction in vitamin D synthesis, hyperphosphatemia, hypocalcemia, and PTH skeletal resistance. ${ }^{3,4}$ This state of mineral metabolism imbalance is independently associated with increased risk of mortality from cardiovascular disease and morbidity, such as bone disease and fractures, among patients with end-stage renal disease on maintenance hemodialysis. ${ }^{5-9}$ Vitamin D replacement therapy with alfacalcidol ( $1 \alpha$-hydroxyvitamin D3) is widely used in the treatment of secondary hyperparathyroidism in hemodialysis patients. Prior studies have shown that alfacalcidol, whether administered orally
Correspondence: Mujahed Shraim Department of Public Health, College of Health Sciences, Qatar University, P.O. Box 2713, Doha, Qatar Tel +974 44037503

Fax+9744403480।

Email mshraim@qu.edu.qa 
or intravenously, is effective in suppressing PTH secretion without significant increase in the risk of hypercalcemia and hyperphosphatemia. ${ }^{10,11}$ Additionally, prior studies have demonstrated that pulse alfacalcidol therapy is effective in suppressing PTH as daily oral alfacalcidol therapy. ${ }^{12,13}$ However, some studies recommend the use of pulse oral alfacalcidol in hemodialysis patients for its ease of administration during hemodialysis sessions, treatment compliance, and cost-effectiveness advantages. ${ }^{14,15}$

Until now, only very few studies have compared the effects of pulse oral alfacalcidol therapy versus daily oral alfacalcidol therapy in suppressing PTH among hemodialysis patients. ${ }^{12,13}$ One study only included a relatively small number of hemodialysis patients (16 patients in the daily group and 18 patients in the pulse group). Also, the findings of these studies regarding the effectiveness of pulse oral alfacalcidol therapy versus daily oral alfacalcidol therapy in suppressing PTH were unadjusted and did not account for the independent effects of various demographic and biomedical characteristics of hemodialysis patients on PTH levels (for example, age, duration on hemodialysis, diabetic status), which were reported as significant predictors of PTH levels among hemodialysis and peritoneal dialysis patients. ${ }^{16}$ Identifying demographic and biomedical characteristics that independently affect PTH levels among hemodialysis patients is important. Such information can be used in clinical practice in developing individualized treatment plans for hemodialysis patients, which may help in achieving optimal target ranges for PTH and maintain mineral metabolism balance. The aim of this randomized controlled trial was to replicate the findings of previous studies comparing effectiveness of pulse oral alfacalcidol therapy versus daily oral alfacalcidol therapy in suppressing PTH using a Palestinian sample of hemodialysis patients, and to identify demographic and biomedical patients' characteristics independently associated with PTH levels.

\section{Methods}

\section{Study design, population, and setting}

This randomized controlled study was conducted in the hemodialysis unit at An-Najah National University Hospital, Nablus, Palestine. The study lasted for 13 weeks (October 1, 2016, to January 1, 2017). The Institutional Review Board of College of Medicine and Health Sciences at An-Najah National University granted ethical approval for this study under ethical approval archive number 101/OCT/2015. All patients signed informed consent for participation in the study. Subjects had to meet the following eligibility criteria for inclusion in the study: age $\geq 18$ years old and with mean values from three consecutive readings of $<10.5 \mathrm{mg} / \mathrm{dL}$ for serum corrected calcium, $<8.5 \mathrm{mg} / \mathrm{dL}$ for serum phosphorus, and $>180 \mathrm{pg} / \mathrm{mL}$ for PTH (more than three times the upper normal limit of PTH [60 pg/mL]). Subjects who did not meet the inclusion criteria and those with any concomitant chronic or malignant liver disease were excluded. All patients were on regular three hemodialysis sessions per week (4 hours per session) using a dialysate calcium concentration of $1.25 \mathrm{mmol} / \mathrm{L}$.

\section{Treatment groups}

We used the random number generation function in the Statistical Package for Social Sciences (SPSS, version 23.0, IBM Corp., Armonk, NY, USA) to randomize patients to receive either daily oral dose of alfacalcidol (daily group) or thrice-weekly oral dose of alfacalcidol (pulse group). The alfacalcidol oral tablets preparations available in the unit were $0.25 \mathrm{mcg}$ and $1.0 \mathrm{mcg}$. The alfacalcidol dosage for every patient in both treatment groups was based on baseline serum PTH levels and was adjusted monthly based on laboratory findings as described by KDIGO criteria. ${ }^{23}$ The thrice-weekly alfacalcidol for each patient in the pulse group was calculated using the following formula: thrice-weekly dose = (daily dose $\times 7) / 3$. Then the resulting dosage of alfacalcidol was rounded to the multiples of $0.25 \mathrm{mcg}$. Patients in the daily group received their individualized dosage of alfacalcidol as usual (7 days per week), while patients in the pulse group received their individualized alfacalcidol dosage at the end of each hemodialysis session. Phosphate binders used in the study for all patients were calcium carbonate (600 mg tablet), calcium acetate $700 \mathrm{mg}$ tablet, and Sevelamer hydrochloride $800 \mathrm{mg}$ tablet.

\section{Measurements}

Corrected serum calcium and phosphate were measured at baseline and were repeated on monthly basis, while serum PTH was measured at baseline and at the end of the study. However, any extremes values for corrected serum calcium or phosphate and for serum PTH were repeated after 2 weeks and 1 month, respectively. The alfacalcidol dosage was discontinued if the PTH reading was less than $150 \mathrm{pg} / \mathrm{mL}$, and the serum PTH was repeated next month. Corrected serum calcium level was determined using the formula: albumin-adjusted serum calcium concentration $(\mathrm{mg} / \mathrm{dL})$ $=$ measured serum calcium concentration $(\mathrm{mg} / \mathrm{dL})+0.8$ $\times[4-$ serum albumin concentration $(\mathrm{g} / \mathrm{dL})] \cdot{ }^{24}$ All blood samples from patients were drawn from the dialyzer line 
before starting hemodialysis session. Laboratory serological tests were carried out at An-Najah National University Hospital laboratories.

\section{Statistical analysis}

Descriptive statistics were used to summarize demographic and biomedical characteristics of patients. Frequencies with percentages were used to summarize categorical variables and mean with standard deviation was used for continuous variables. Independent samples $t$-test and $\chi^{2}$ test were used to examine for any statistically significant differences between treatment groups on baseline demographic and biomedical characteristics. Paired-samples $t$-test was used for comparing changes in serum PTH, corrected calcium, and phosphate levels at baseline and at 13 weeks. Repeated measures analyses using a linear mixed-modeling approach were used to estimate effect of independent variables (treatment group [daily, pulse], age, gender [female, male], diabetic status [yes, no], and duration of dialysis in years) on changes in serum PTH, corrected calcium, and phosphate levels at 13 weeks with their respective baseline levels. All tests were two-sided. A $P$-value less than 0.05 was considered statistically significant. All analyses were conducted using SPSS computer program (version 23.0, IBM Corp).

\section{Results}

\section{Baseline characteristics of patients}

One hundred and eighty-four patients were enrolled at the beginning of the study, 93 in the daily group and 91 in the pulse group. Only 167 patients completed the study, 88 in the daily group and 79 in the pulse group. In the daily group, 4 patients died and one patient dropped out for undergoing renal transplantation. Reasons for death in the daily group were due to myocardial infarction (2 patients), colon cancer (1 patient), and heart failure (1 patient). In the pulse group, 6 patients died, one patient dropped out for undergoing renal transplantation, one patient was not compliant with hemodialysis, and four patients decided to withdraw from the study (two of them reported gastrointestinal disturbances). Causes for death in the pulse group were due to cerebrovascular accidents ( 3 patients), sepsis ( 2 patients), and pulmonary embolism (1 patient).

The baseline demographic and biomedical characteristics of patients who completed the study are presented in Table 1 . There were no statistically significant differences between the daily and pulse groups in age; gender; duration on dialysis; diabetic status; serum PTH, corrected calcium, phosphorus levels, and therapeutic doses for alfacalcidol,
Table I Baseline demographic and biomedical characteristics of hemodialysis patients by treatment group

\begin{tabular}{|c|c|c|c|}
\hline Variable & $\begin{array}{l}\text { Daily group } \\
(n=88)\end{array}$ & $\begin{array}{l}\text { Pulse group } \\
(n=79)\end{array}$ & $P$-value \\
\hline Age (years) & $58.26(12.7)$ & $56.84(14.3)$ & 0.498 \\
\hline Gender, n (\%) & & & 0.645 \\
\hline Female & $4 \mid(46.6)$ & $34(43.0)$ & \\
\hline Male & $47(53.4)$ & $45(57.0)$ & \\
\hline Diabetes status, n (\%) & & & 0.524 \\
\hline Diabetic & $50(56.8)$ & $4 \mid(5 I .9)$ & \\
\hline Nondiabetic & $38(43.2)$ & $38(48.1)$ & \\
\hline Duration on dialysis (years) & $3.7(3.2)$ & $3.7(3.5)$ & 0.976 \\
\hline Serum PTH pg/mL & $528.4(550.8)$ & $552.4(467.5)$ & 0.762 \\
\hline Serum corrected calcium mg/dL & $8.9(0.9)$ & $8.9(0.9)$ & 0.831 \\
\hline Serum phosphate $\mathrm{mg} / \mathrm{dL}$ & $5.2(1.5)$ & $5.3(1.56)$ & 0.665 \\
\hline Alfacalcidol tablets (mcg) & $0.7(0.5)$ & $0.8(0.6)$ & 0.440 \\
\hline Calcium tablets (mg) & $2.6(2.3)$ & $2.2(2.1)$ & 0.212 \\
\hline Calcium acetate tablets $(\mathrm{mg})$ & $0.7(1.6)$ & $1.0(1.9)$ & 0.337 \\
\hline Sevelamer tablets $(\mathrm{mg})$ & $0.78(1.4)$ & $1.2(2.1)$ & 0.151 \\
\hline
\end{tabular}

Notes: Data are mean (SD), unless otherwise stated.

Abbreviations: PTH, parathyroid hormone; SD, standard deviation.

calcium carbonate and calcium acetate, and Sevelamer hydrochloride.

\section{Therapeutic efficacy}

At the end of the study, PTH levels were lower in both treatment groups as compared with baseline levels (Table 2). The mean reduction in PTH from baseline in the daily group was $43 \mathrm{pg} / \mathrm{mL}$, but this was not statistically significant $(P=0.159)$. However, the mean reduction in PTH from baseline in the pulse group was $118 \mathrm{pg} / \mathrm{mL}$ and statistically significant $(P<0.001)$; Figure 1 .

After 13 weeks of therapy, there were no statistically significant differences between the two groups in proportions of patients reaching therapeutic end point for PTH level (150-300 pg/mL). The therapeutic end point for PTH levels was roughly equal (38\%) in both groups. Similarly, at 13 weeks, roughly equal proportions (9\% and 53\%) of patients in both groups had PTH levels $<150 \mathrm{pg} / \mathrm{mL}$ and $>300 \mathrm{pg} / \mathrm{mL}$, respectively. However, higher proportion of patients in the pulse group $(39.2 \%)$ had $30 \%$ or more reduction in PTH during the study than the daily group (26.4\%), which is close to being statistically significant $\left(\chi^{2}(1)=3.7, P=0.054\right)$.

\section{Effects on metabolism of calcium and phosphate}

At 13 weeks, there were no statistically significant differences in mean corrected serum calcium levels between treatment groups, and their mean values remained within the normal 
range (Table 2). However, mean corrected serum calcium increased slightly in the pulse group and dropped slightly in the daily group (Table 2; Figure 2). Similarly, we found no statistically significant differences in mean serum phosphate levels between treatment groups (Table 2). The mean serum phosphate levels dropped slightly in a roughly identical way in both groups at 13 weeks and their mean values remained within the normal range (Table 2; Figure 3).

\section{Factors associated with changes in PTH levels at I 3 weeks}

After controlling for demographic and biomedical characteristics of patients, the results of repeated measures analysis using linear mixed models for PTH levels showed that the mean reduction in serum PTH levels at 13 weeks was lower in the pulse group than the daily group by $44 \mathrm{pg} / \mathrm{mL}$, but this difference was not statistically significant (Table 3). As shown in Table 3 , age and diabetic status were independently associated with changes in serum PTH levels at 13 weeks with the respective baseline levels. Increase in age by 1 year was associated with decrease in mean serum PTH by $6.7 \mathrm{pg} /$ $\mathrm{dL}$ with the respective baseline levels $(P=0.022)$. At the end of the study, diabetic patients had significant reduction in mean serum PTH levels by 166 pg/dL (P.037).

\section{Occurrence of side effects}

Only two patients in the pulse group reported gastrointestinal disturbances. No side effects were reported in the daily group.

Three patients (3.8\%) in the pulse group and 5 patients $(5.7 \%)$ in the daily group developed hypercalcemia (corrected serum calcium $>10.5 \mathrm{mg} / \mathrm{dL}$ ) by the end of the study, but this was not statistically significant. Similarly, there was no statistically significant difference between the two treatment groups in occurrence of hyperphosphatemia (serum phosphate $>4.5 \mathrm{mg} / \mathrm{dL}$ ); but hyperphosphatemia occurred in 49 patients $(62.0 \%)$ in the pulse group and in 53 patients $(60.2 \%)$ in the daily group during the study.

\section{Evaluation of compliance}

All patients in the pulse group received their individualized alfacalcidol doses by nursing staff at the end of their hemodialysis sessions during the study period. Patients in the daily group were instructed to intake their alfacalcidol doses 7 days a week as usual, but we have no information about compliance with alfacalcidol therapy in this group. The staff did regular frequent follow-ups to ensure patients compliance to their doses as much as possible during the period of the study. 


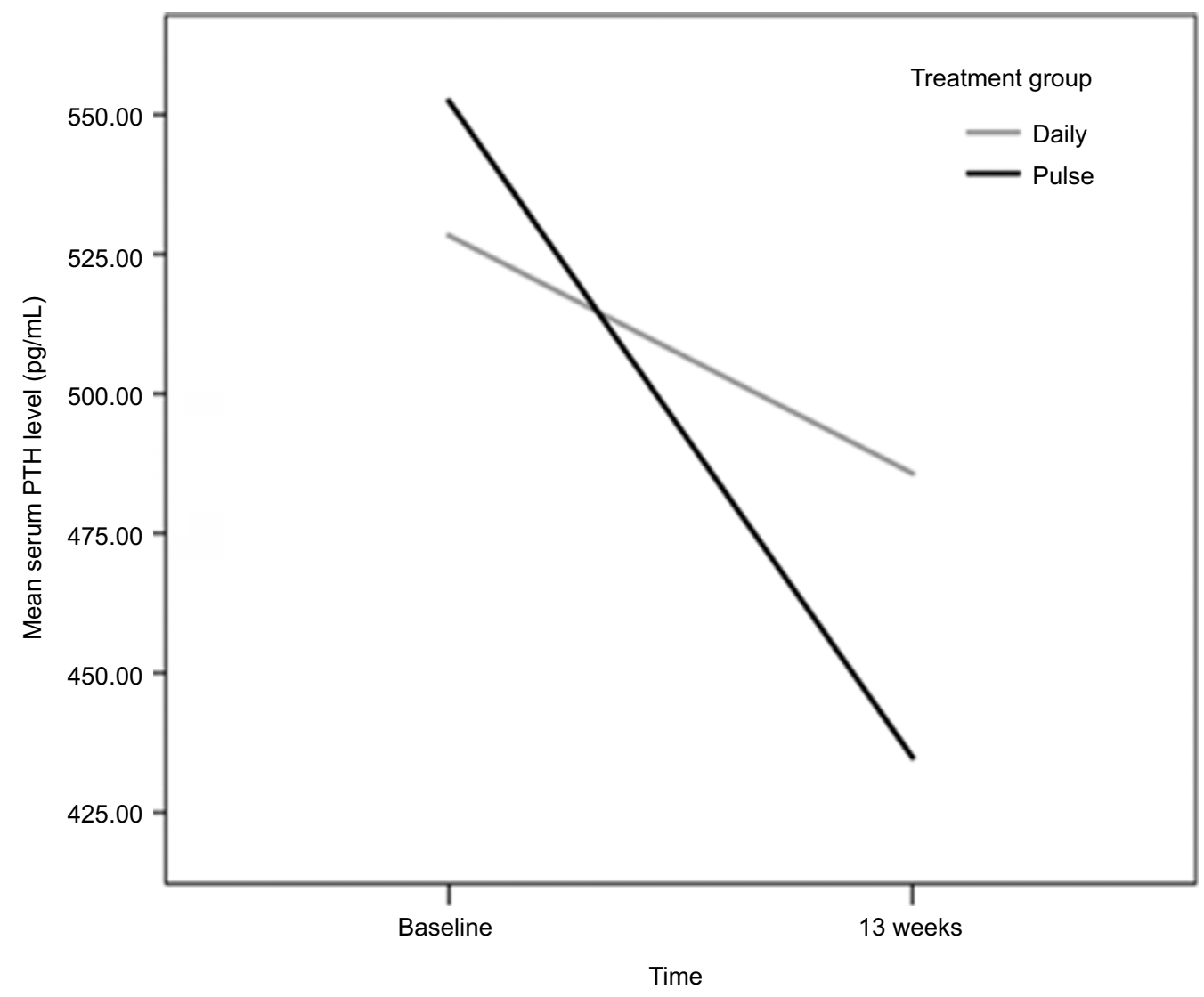

Figure I Mean PTH levels at baseline and at 13 weeks.

Abbreviation: PTH, parathyroid hormone.

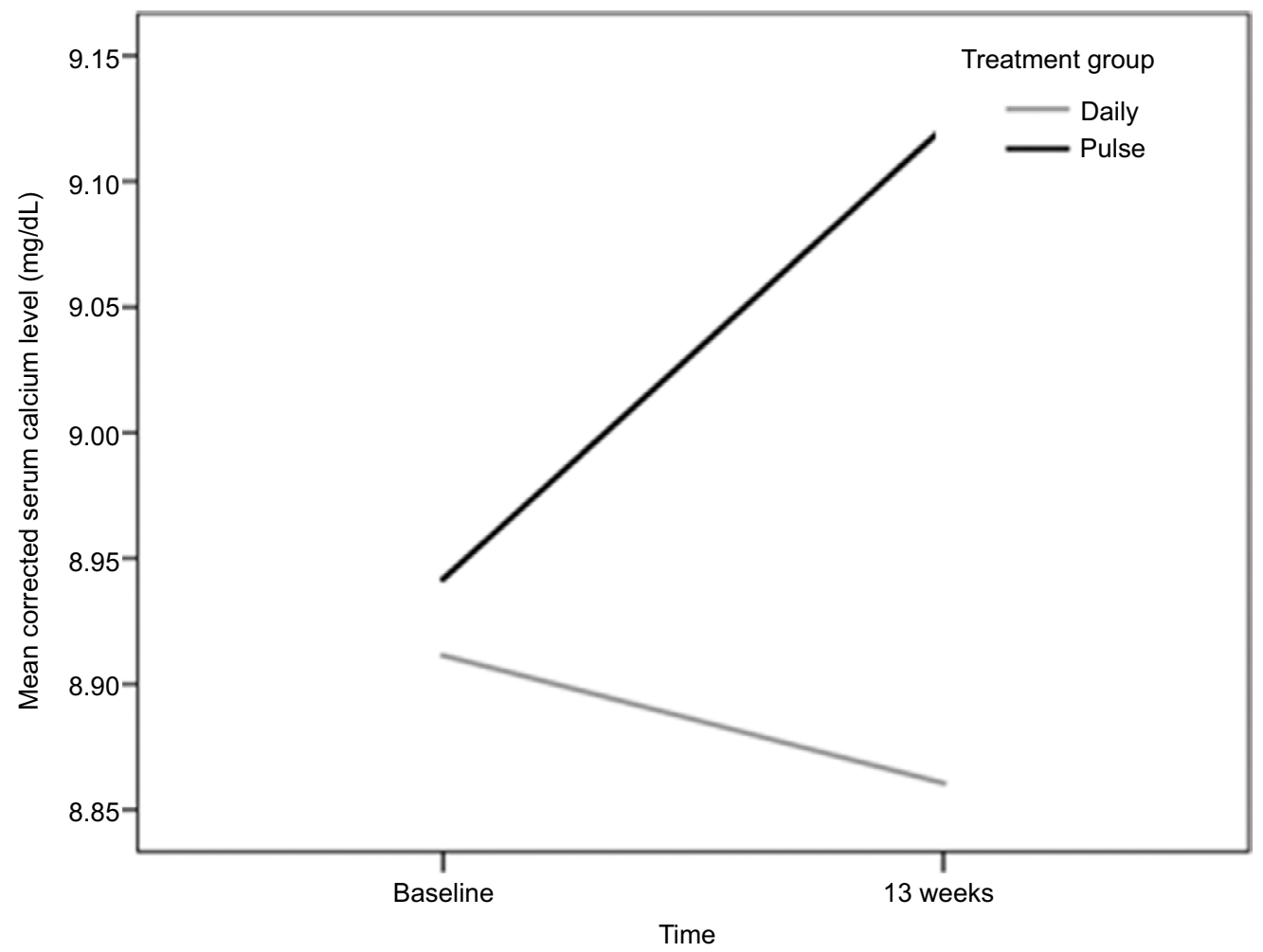

Figure 2 Mean corrected serum calcium levels at baseline and at 13 weeks. 


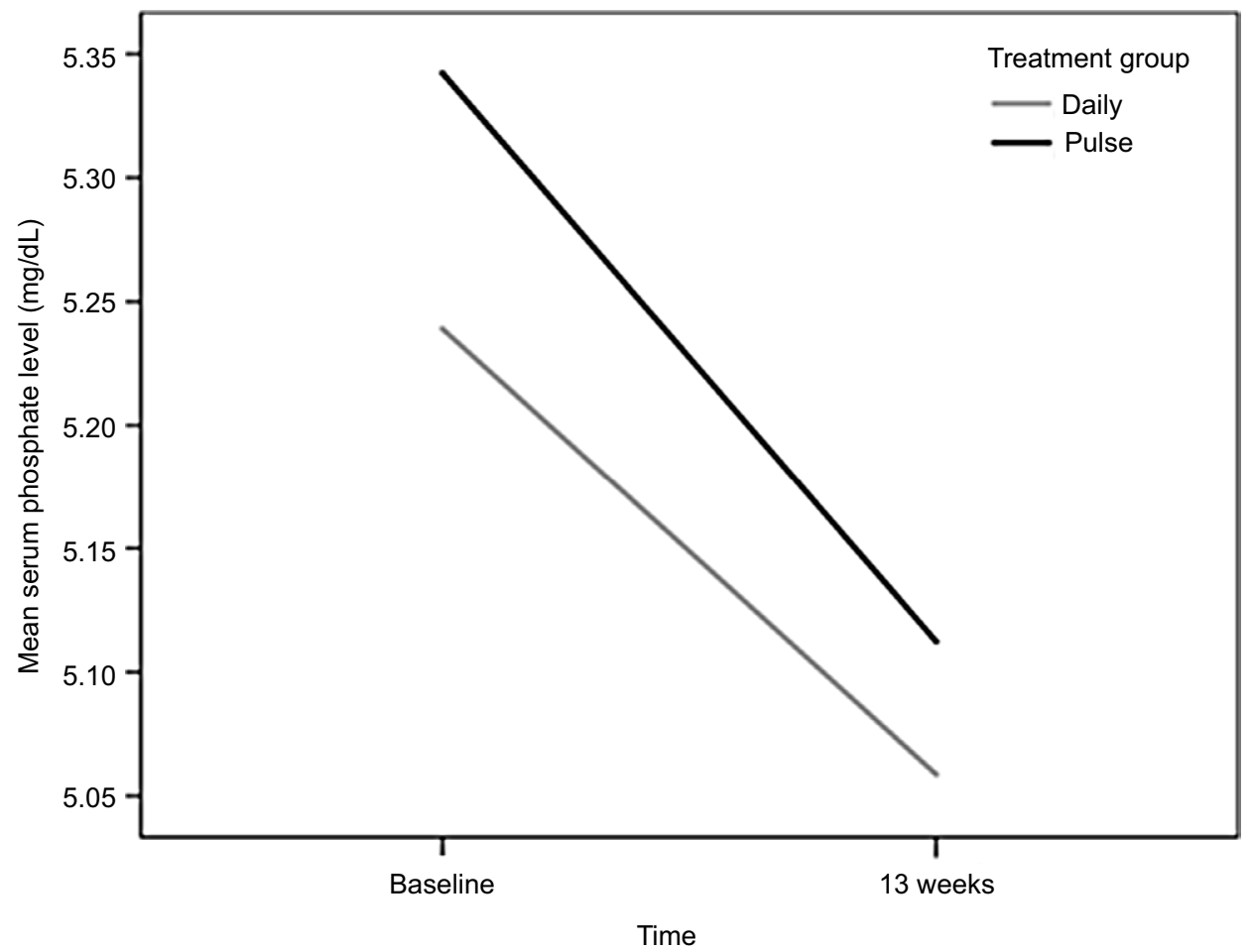

Figure 3 Mean phosphate levels at baseline and at 13 weeks.

Table 3 Factors associated with changes in mean PTH levels at 13 weeks

\begin{tabular}{llll}
\hline Variable & Effect estimate & SE & P-value \\
\hline $\begin{array}{l}\text { Treatment group } \\
\quad \text { Daily }\end{array}$ & Reference category & 71.3 & 0.539 \\
$\quad \begin{array}{l}\text { Pulse } \\
\text { Age }\end{array}$ & -44.0 & 2.9 & 0.022 \\
$\begin{array}{l}\text { Gender } \\
\quad \text { Female }\end{array}$ & -6.7 & & 0.995 \\
$\quad$ Rale & 0.5 & 72.3 & \\
$\begin{array}{l}\text { Diabetic status } \\
\quad \text { Nondiabetic }\end{array}$ & Reference category & & 0.037 \\
$\quad$ Diabetic & -166.4 & 79.0 & \\
$\begin{array}{l}\text { Duration on dialysis } \\
\text { (years) }\end{array}$ & 22.4 & 11.4 & 0.051 \\
\hline Abriation & & & \\
\hline
\end{tabular}

Abbreviations: PTH, parathyroid hormone; SE, standard error.

\section{Discussion}

The aim of this randomized controlled trial was to compare effectiveness of pulse oral alfacalcidol therapy versus daily oral alfacalcidol therapy in suppressing PTH and identify demographic and biomedical patients' characteristics independently associated with PTH levels. There were no statistically significant differences between the two treatment groups in baseline demographic and biomedical characteristics. Both groups had decreased mean PTH levels at the end of the study. The pulse group had more clinically significant reduction in mean PTH level at 13 weeks than the daily group, but this reduction was not statistically significant. The effect of alfacalcidol therapy on metabolism of phosphate at the end of the study, characterized by slight reduction in mean phosphate levels, was similar in both groups. The mean corrected serum calcium increased slightly in the pulse group and dropped slightly in the oral group at the end of the study, but remained within normal range in both groups. These findings are consistent with prior studies and provide further support that pulse oral alfacalcidol therapy is as effective in suppressing PTH levels as daily oral alfacalcidol therapy without significant increase in the risk of hypercalcemia or hyperphosphatemia, with the additive advantage of ensuring complete compliance with therapy. ${ }^{12,13}$

This study showed that mean PTH levels were inversely associated with older age and being diabetic. This finding is also consisted with previous studies that observed lower PTH levels among diabetics ${ }^{16-18}$ and older hemodialysis patients. ${ }^{16}$ The exact mechanism underlying this is not well understood, but it was hypothesized to be explained by different factors, including malnutrition, inflammation, ${ }^{16,19}$ and poor glycemic control in diabetic patients. ${ }^{16}$ Lower serum PTH levels among diabetic patients may suggest that diabetic patients on hemodialysis might be at greater risk of cardiovascular mortality and morbidity than nondiabetic patients. Low serum PTH in hemodialysis patients has been linked to increase risk of cardiovascular mortality. ${ }^{16,20,21}$ 


\section{Strengths and limitations}

This study included relatively a large number of patients undertaking hemodialysis in An-Najah National University Hospital. The number of hemodialysis patients in this unit represents about $20 \%$ of all hemodialysis patients' population in West Bank, Palestine. ${ }^{22}$ So, the sociodemographic and biomedical characteristics of enrolled patients are highly likely representative of the hemodialysis population in West Bank, Palestine. Additionally, compliance with alfacalcidol therapy in the pulse group was complete during the study as patients received their pulse dose of alfacalcidol at the end of each dialysis session and this was given by nursing staff in the hemodialysis unit.

One of the limitations of this study is that we were unable to use a precise measurement for compliance with alfacalcidol therapy in the daily group. However, all patients in the daily group were instructed to intake their medications as usual 7 days a week. So, it is unlikely that noncompliance with medications in the daily group has affected the magnitude and direction of alfacalcidol effects on mean PTH levels significantly as our findings are very consistent with the findings of prior studies.

\section{Conclusion}

This study adds further evidence that pulse oral alfacalcidol therapy is as effective in suppressing PTH levels as daily oral alfacalcidol therapy, without the significant increase in the risk of hypercalcemia or hyperphosphatemia. For clinicians treating hemodialysis patients with alfacalcidol, switching daily alfacalcidol therapy to thrice-weekly alfacalcidol pulse therapy seems safe and convenient, especially for hemodialysis patients with poor compliance with treatment. This study also suggests that monitoring and prevention of malnutrition with optimal glycemic control in diabetic patients may help in minimizing the risk of mortality from cardiovascular disease in patients with lower levels of serum PTH.

\section{Availability of data and materials}

Data are all contained within the article. The raw data are available with the corresponding author and can be given on requested.

\section{Acknowledgment}

We thank the participants and nursing staff in the hemodialysis unit at An-Najah National University Hospital for making the study possible.

\section{Author contributions}

$\mathrm{ZH}, \mathrm{MS}$, OS, SM, KA, EK, and HM designed the study and its protocol. OS, SM, and HM collected the data. HM arranged the pharmaceutical and medication issues. OS, SM, ZH, KA, HM, and EK managed follow-up of participants. MS performed data analysis. All authors interpreted the data, reviewed the manuscript critically for important intellectual content, and read and approved the final manuscript for submission.

\section{Disclosure}

The authors report no conflict of interest in this work.

\section{References}

1. Cannata-Andía JB, Martin KJ. The challenge of controlling phosphorus in chronic kidney disease. Nephrol Dial Transplant. 2016;31: $541-547$.

2. Cannata-Andía JB, Carrera F. The pathophysiology of secondary hyperparathyroidism and the consequences of uncontrolled mineral metabolism in chronic kidney disease: the role of COSMOS. NDT Plus. 2008;1:i2-i6.

3. Portillo MR, Rodríguez-Ortiz ME. Secondary hyperparthyroidism: pathogenesis, diagnosis, preventive and therapeutic strategies. Rev Endocr Metab Disord. 2017;18:79-95.

4. Rodriguez M, Canalejo A, Garfia B, Aguilera E, Almaden Y. Pathogenesis of refractory secondary hyperparathyroidism. Kidney Int Suppl. 2002;(80):155-160.

5. Komaba H, Igaki N, Takashima M, et al. Calcium, phosphorus, cardiovascular events and all-cause mortality in hemodialysis patients: a singlecenter retrospective cohort study to reassess the validity of the Japanese Society for Dialysis Therapy guidelines. Ther Apher Dial. 2008;12: 42-48.

6. Block GA. Therapeutic interventions for chronic kidney disease-mineral and bone disorders: focus on mortality. Curr Opin Nephrol Hypertens. 2011;20:376.

7. Block GA, Klassen PS, Lazarus JM, Ofsthun N, Lowrie EG, Chertow GM. Mineral metabolism, mortality, and morbidity in maintenance hemodialysis. J Am Soc Nephrol. 2004;15:2208-2218.

8. Arici M, Kahraman S, Gençtoy G, et al. Association of mineral metabolism with an increase in cellular adhesion molecules: another link to cardiovascular risk in maintenance haemodialysis? Nephrol Dial Transplant. 2006;21:999-1005.

9. Kalantar-Zadeh K, Kuwae N, Regidor DL, et al. Survival predictability of time-varying indicators of bone disease in maintenance hemodialysis patients. Kidney Int. 2006;70:771-780.

10. Kandula P, Dobre M, Schold JD, Schreiber MJ, Mehrotra R, Navaneethan SD. Vitamin D supplementation in chronic kidney disease: a systematic review and meta-analysis of observational studies and randomized controlled trials. Clin J Am Soc Nephrol. 2011;6: $50-62$

11. Lee WT, Padayachi K, Collins JF, Cundy T. A comparison of oral and intravenous alfacalcidol in the treatment of uremic hyperparathyroidism. J Am Soc Nephrol. 1994;5:1344-1348.

12. Tarrass F, Yazidi A, Sif H, Zamd M, Benghanem MG, Ramdani B. A randomized trial of intermittent versus continuous oral alfacalcidol treatment of hyperparathyroidism in end-stage renal disease. Clin Nephrol. 2006;65:415-418.

13. Gu Y, Ding F, Chen N, et al. Comparisons between oral pulse alfacalcidol therapy and daily therapy in maintenance hemodialysis patients with secondary hyperparathyroidism: a randomized, controlled, and multicenter study. Renal Fail. 2005;27:205-212.

14. Lessard M, Ouimet D, Leblanc M, et al. Comparison of oral and intravenous alfacalcidol in chronic hemodialysis patients. BMC Nephrol. 2014;15:27.

15. Mitwalli AH, Alam AA. Intermittent oral versus intravenous alfacalcidol in dialysis patients. Saudi J Kidney Dis Transpl. 2000;11 174-180. 
16. Avram MM, Mittman N, Myint MM, Fein P. Importance of low serum intact parathyroid hormone as a predictor of mortality in hemodialysis and peritoneal dialysis patients: 14 years of prospective observation. Am J Kidney Dis. 2001;38:1351-1357.

17. Inaba $M$, Nagasue $K$, Okuno $S$, et al. Impaired secretion of parathyroid hormone, but not refractoriness of osteoblast, is a major mechanism of low bone turnover in hemodialyzed patients with diabetes mellitus. Am J Kidney Dis. 2002;39:1261-1269.

18. Inaba M, Okuno S, Nagasue K, et al. Impaired secretion of parathyroid hormone is coherent to diabetic hemodialyzed patients. Am J Kidney Dis. 2001;38:S139-S142.

19. Gnudi L. Serum intact parathyroid hormone in diabetic patients on haemodialysis: what is the treatment goal? Nephrol Dial Transplant. 2008;23:24-26.

20. Villa-Bellosta R, Rodriguez-Osorio L, Mas S, et al. A decrease in intact parathyroid hormone (iPTH) levels is associated with higher mortality in prevalent hemodialysis patients. PLoS One. 2017;12:e173831.
21. Drechsler C, Krane V, Grootendorst DC, et al; German Diabetes and Dialysis Study Investigators. The association between parathyroid hormone and mortality in dialysis patients is modified by wasting. Nephrol Dial Transplant. 2009;24:3151-3157.

22. Palestinian Health Information Center: Health annual report: Palestine 2015. Ramallah, Palestinian Health Information Center, 2016. Available from: http://www.moh.ps/Content/Books/kD3bquHr7jbwK9f6VQJAsLDCuckgED1CZUFa9ssb62m9Eim21e562D_ECDSNEboZRJwc6HyiggSMzKUPMeDJa2vkBN1AdZOGlvNuS9 CHKJjGO.pdf.

23. Kidney Disease International Global Outcomes. KDIGO 2012 Clinical Practice Guideline for the Evaluation and Management of Chronic Kidney Disease. Kidney Int Suppl. 2013;3(1):1-150.

24. Becerra-Tomás N, Estruch R, Bulló M, et al. Increased serum calcium levels and risk of type 2 diabetes in individuals at high cardiovascular risk. Diabetes Care. 2014;37:3084-3091.

\section{Publish your work in this journal}

The International Journal of Nephrology and Renovascular Disease is an international, peer-reviewed open access journal focusing on the pathophysiology of the kidney and vascular supply. Epidemiology, screening, diagnosis, and treatment interventions are covered as well as basic science, biochemical and immunological studies. The manuscript management system is completely online and includes a very quick and fair peer-review system, which is all easy to use. Visit http://www. dovepress.com/testimonials.php to read real quotes from published authors. 